nate, the source should be sought for in internal hæmorrhage or in intracranial disturbance ; if the thoracic symptoms are most prominent, the air passages or the circulatory apparatus are to be questioned, and the former can most readily be excluded. The only positive evidence in favor of the latter is to be derived from the previous history of the patient.

\title{
A MODIFICATION OF THE OPHTHALMOSCOPE.1
}

BY O. F. WADSWORTH, M. D.

DURing the last few years the direct method of ophthalmoscopic examination (upright image) has come more and more into favor. While it enables the inspection of the background of the eve under a much greater magnifying power than by the indirect method, and is free from the disturbing reflections from the surfaces of the auxiliary lens which attend the latter, it also permits the tolerably close determination of the refraction of the observed eye independently of the subjective impressions of the patient. For the patient the direct method has the important advantage that a far less amount of light need be thrown into his eye, and the often disagreeable dazzling caused by the indirect method is thus avoided.

To the generalization of this method the modification of Loring, which consisted in placing a rotating disk containing lenses of different focal power behind the mirror, has chiefly contributed. In his ophthalmoscope, as in nearly all the various modifications of it which have since appeared, the mirror stands in a plane parallel to that of the disk behind it. To this there is an objection. With such an arrangement, in order to throw the light from the lamp into the observed eye, the mirror, and also the correcting lens behind it, must be placed at an angle to the direction in which the observer looks and which the rays coming from the fundus of the patient's eye to enter his follow. The effect of this oblique position of the lens on the rays which traverse it is as if there were added to it a cylindrical lens, its axis corresponding to the axis on which the spherical lens is inclined. The lens is made practically astigmatic, and the image of the fundus seen through it is distorted as it would be if the observed eye were astigmatic. The degree of astigmatism increases with the increase of obliquity of the lens, and for a given degree of obliquity is proportionate to the strength of the lens. When the mirror is placed in suitable proximity to the observed eye, an inclination of $20^{\circ}$ is as little as can well be given to it in order to reflect the light into the eye in such a way that the fundus may be seen. For the lower degrees of ametropia the amount of astigmatism caused

1 Read before the Boston Society of Medical Sciences, December 26, 1876. 
by such an inclination of the correcting lens is so small that it may in practice be neglected. But when a lens of high power is needed behind the mirror, that is, with a high degree of ametropia of the observed or observing eye, or of both combined, the astigmatism becomes very considerable (with a lens of $\frac{1}{2}$ amounting to as much as $\mathrm{Y}^{1} \mathrm{f}$ ), and no accurate determination of the refraction can be made. Nor can a distinct image of the fundus of an eye with a high degree of ametropia be thus obtained. This latter fact is generally recognized, though the cause is not so generally known.

There are, however, ophthalmoscopes with the mirror inclined to the plane in which the correcting lenses lie, Jaeger's being the most familiar, and to these the above objection does not apply. But in all these, so far as I have been able to obtain knowledge of them, the mirror is so placed that its central opening is at a very considerable distance in front of the correcting lens, and this entails the disadvantage that it contracts the field of view of the observer, and in case the observed eye be hypermetropic it diminishes the amount of light received by him.

The modification which I wish to describe is designed to correct

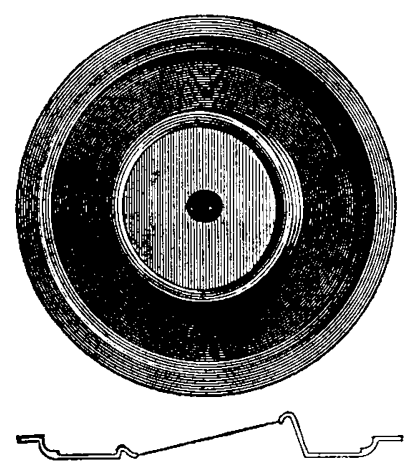
both the defects mentioned. A concave mirror of seven inches focus and fifteen $\mathrm{mm}$. in diameter, is set at an angle of $20^{\circ}$ in the centre of a thin plate which takes the place of the ordinary mirror in a Loring's ophthalmoscope. By thus diminishing the size of the mirror it has been possible to set it so far back that the hole in its centre, $4 \mathrm{~mm}$. in diameter, is at its farthest edge only $4 \mathrm{~mm}$. from the anterior surface of the correcting lens behind it. The plate with the mirror rotates, so that the latter may be turned toward the right or left and the instrument be used for either eye. The small size of the mirror offers no impediment to examination by the direct method, since by this method, on account of the nearness of the mirror to the observed eye, only the light reflected from the parts of the mirror immediately about the central perforation can enter the pupil. The small mirror does not, however, give light enough for the indirect method, and it is therefore arranged so that it may be readily detached and a full-sized mirror substituted. But where the ophthalmoscope is in frequent use it will be found more convenient to have a second ophthalmoscope, to be used for the reversed image only, with which a movable clip containing a $10^{\prime \prime}$ or $12^{\prime \prime}$ convex lens belind the mirror would be sufficient. The large size of the opening in the mirror, $4 \mathrm{~mm}$., offers an advantage in determining errors of refraction, provided the pupil be large, since where the refraction is im- 
perfectly corrected it allows the formation of larger circles of dispersion on the retina of the observer, and hence the imperfection of the correction may be more easily recognized. The instrument to which $I$ have had this mirror fitted is one of the earlier pattern of loring, with three removable disks. The lenses in the later patterns of Loring and in most other " refraction" ophthalmoscopes are too small to admit the use of so large a hole in the mirror with advantage, and with these a smaller hole may be used.

The mirror may be obtained of Mr. H. W. Hunter, optician, 1132 Broadway, New York.

\section{RECENT PROGRESS IN PHYSIOLOGY.1}

BY HENRY P. BOWDITCh, M. D.

PERCEPTION OF TONES AND NOISES.

EXNER ${ }^{2}$ discusses the question whether we cannot suppose that the organ of Corti serves for the perception of noises as well as of musical tones instead of assuming with Helmholtz ${ }^{3}$ that its use is limited to the latter purpose only, and that noises (that is, a single sonorous impulse or an irregular series of them) are heard by means of the nerve terminations in the vestibular sac and in the ampullæ. The question derives additional interest from the fact that the semicircular canals and the ampullæ are now almost universally regarded as organs serving to the maintenance of equilibrium. Even to the utriculus a function of this sort has been assigned, leaving only the sacculus hemisphericus for the perception of noises, unless it can be supposed that the same organ may serve for two such totally different functions as hearing and the preservation of equilibrium. Exner first endeavors to determine whether there is any good reason to suppose that noises may not be perceived by the organ of Corti. To produce a single sonorous vibration he makes use of the snap of an electric spark which has been shown by Töpler ${ }^{4}$ to give rise to only one sound-wave. Now it is found that two electric sparks following each other at an interval of only $0.002^{\prime \prime}$ are recognized by the ear as not absolutely synchronous. If this brief interval is recognized by means of Corti's organ we must suppose that certain fibres are set in vibration by the first sound-wave and that before the second wave reaches them, that is, in $0.002^{\prime \prime}$, time enough has elapsed for the amplitude of the vibration to become perceptibly diminished. Now, it has been shown by Helmholtz that the fibres in the cochlea are so constituted that when left to themselves after being set

1 Concluded from page 76.

2 Pflüger's Archiv, xiii. 228, and Centralblatt, 1876, 756.

- Tonempfindungen, iii. Aufl. page 226.

* Beobachtungen nach einer neuen optischen Methode. Bonn. 1864. 